\title{
Prevalence, incidence and persistence of ADHD drug use in Japan
}

Received 4 February 2018; Accepted 28 April 2018; First published online 28 May 2018

Key words: ADHD, adolescent health, atomoxetine, methylphenidate, pharmacotherapy.

\section{Introduction}

There are significant geographical variations in the prevalence of attention-deficit/hyperactivity disorder (ADHD) drug use in children and adolescents (Kovess et al. 2015; Beau-Lejdstrom et al. 2016; Burcu et al. 2016; Piovani et al. 2016; Furu et al. 2017; Wang et al. 2017), although the prevalence of ADHD does not vary as a function of geographical location (Thomas et al. 2015). Understanding the geographical discrepancy in drug use will provide insights on potential over- or undermedication in the population. However, little is known about ADHD drug use in the Japanese population. Thus, we aimed to estimate the prevalence, incidence and persistence of ADHD drug use in children and adolescents in Japan.

\section{Methods}

\section{Data source}

A retrospective cohort study was conducted using the National Database of Health Insurance Claim Information and Specified Medical Checkups (NDB) that covered all electronically issued claims covered by public health insurance in Japan (Ministry of Health, Labour \& Welfare, 2013; Okumura et al. 2017). As of April 2014, the proportion of electronically issued claims relative to all claims was $99.9 \%$ in hospitals, $95.9 \%$ in clinics and $99.9 \%$ in pharmacies. Claims for recipients of public assistance were not included in the NDB, comprising 286048 inhabitants aged 0-19 years in 2014 (approximately 1\% of the population). The NDB included information on clinical and procedural characteristics such as patient identification numbers, sex, age group, prescription date, drug codes, days of drug supply and dosage.

* Address for correspondence: Y. Okumura, Department of Psychiatry and Behavioral Science, Tokyo Metropolitan Institute of Medical Science, 2-1-6 Kamikitazawa, Setagaya-ku, Tokyo 156-8506, Japan.

(Email: yokumura@blue.zero.jp)

\section{Settings}

Japan has 21001000 inhabitants aged $\leq 18$ years (Ministry of Internal Affairs \& Communications, 2015). Atomoxetine (ATX) (available since June 2009) and the osmotic-controlled release oral delivery system methylphenidate (OROS-MPH) (available since December 2007) were the only drugs approved for ADHD in the fiscal year of 2014 (between April 2014 and March 2015). OROS-MPH can be prescribed by only licensed physicians with expertise in ADHD treatment. However, immediate-release methylphenidate (IR-MPH) is not approved for ADHD treatment in Japan.

In general, the universal health insurance system of Japan pays for $70 \%$ of the medical treatment costs and the other healthcare systems (i.e. the System of Medical Payment for Services and Supports for Persons with Disabilities; and the Medical Subsidy for Children and Infants) pay for the remaining 20-30\% for children and adolescents.

\section{Statistical analyses}

We identified patients aged $\leq 18$ years who were given at least one prescription of ADHD drug in the fiscal year of 2014. The annual prevalence of ADHD drug use was defined as the number of prevalent users per 1000 inhabitants.

Next, we identified the subgroup focusing on the incident and persistent users of ADHD drugs. First, we defined the index date as the date on which the ADHD drug was first prescribed to the patient during the fiscal year of 2014. We included the patients who had been enrolled in the database at least 180 days before and after the index date, as in previous studies (Lawson et al. 2012; Palli et al. 2012). Second, we excluded those who had a bundled payment claim within 180 days before and after the index date, in which the prescription status was not recorded. Finally, we excluded those who had a prescription of ADHD drugs within 180 days before the index date. All patients were followed up using an identification number (Kubo et al. 2018). The annual incidence of ADHD drug use was defined as the

This is an Open Access article, distributed under the terms of the Creative Commons Attribution licence (http://creativecommons.org/licenses/by/ $4.0 /)$, which permits unrestricted re-use, distribution, and reproduction in any medium, provided the original work is properly cited. 
number of incident users per 1000 inhabitants. The percentage of persistent ADHD drug use was defined as the number of persistent users at least 150 days after the index date per 100 incident users, as in a previous study (Lawson et al. 2012). Discontinuation (nonpersistence) was designated when a prescription medication for ADHD was not refilled within an interval defined by the days of drug supply plus a grace period of 30 days.

Subgroup analyses were conducted by sex and age groups. Age was classified into four groups: 0-6, 7$12,13-15$ and 16-18 years according to the school system (preschool, elementary school, junior high school and high school, respectively). All estimates were calculated with $95 \%$ confidence intervals (CI). All analyses were conducted using $\mathrm{R}$ version 3.4.1.

\section{Results}

There were 86756 prevalent and 30449 incident users of ADHD drugs in the database (Table 1). The annual prevalence per 1000 inhabitants was 4.1 with a peak of 7-12 years for both sexes (Table 2). Of the prevalent

Table 1. Sample characteristics of patients

\begin{tabular}{|c|c|c|c|c|}
\hline \multirow[b]{2}{*}{ Characteristic } & \multicolumn{2}{|c|}{$\begin{array}{l}\text { Prevalent user } \\
(N=86756)\end{array}$} & \multicolumn{2}{|c|}{$\begin{array}{l}\text { Incident user } \\
(N=30449)\end{array}$} \\
\hline & $N$ & $\%$ & $n$ & $\%$ \\
\hline \multicolumn{5}{|l|}{ Provider type } \\
\hline Clinic & 41500 & 47.8 & 15055 & 49.4 \\
\hline Hospital & 45256 & 52.2 & 15394 & 50.6 \\
\hline \multicolumn{5}{|l|}{ Setting } \\
\hline Inpatient & 508 & 0.6 & 220 & 0.7 \\
\hline Ambulatory & 86248 & 99.4 & 30229 & 99.3 \\
\hline \multicolumn{5}{|l|}{ Prescriber } \\
\hline Non-psychiatrist & 46157 & 53.2 & 15239 & 50.0 \\
\hline Psychiatrist & 40599 & 46.8 & 15210 & 50.0 \\
\hline \multicolumn{5}{|l|}{ Sex } \\
\hline Boys & 72520 & 83.6 & 24528 & 80.6 \\
\hline Girls & 14236 & 16.4 & 5921 & 19.4 \\
\hline \multicolumn{5}{|l|}{ Age, years } \\
\hline $0-6$ & 2659 & 3.1 & 2382 & 7.8 \\
\hline $7-12$ & 52626 & 60.7 & 19494 & 64.0 \\
\hline $13-15$ & 22265 & 25.7 & 6051 & 19.9 \\
\hline $16-18$ & 9206 & 10.6 & 2522 & 8.3 \\
\hline \multicolumn{5}{|c|}{ Individual ADHD drugs } \\
\hline ATX & 31491 & 36.3 & 12876 & 42.3 \\
\hline OROS-MPH & 51013 & 58.8 & 17384 & 57.1 \\
\hline Both & 4252 & 4.9 & 189 & 0.6 \\
\hline
\end{tabular}

ADHD, attention-deficit/hyperactivity disorder; ATX, atomoxetine; OROS-MPH, osmotic-controlled release oral delivery system methylphenidate. users, $64 \%$ used OROS-MPH (Table 1). Of the incident users, $61 \%$ still continued drug treatment at 150 days after the index date (Fig. 1). The persistence rate was much higher among those aged 7-12 years than among those aged 16-18 years (65 v. 43\%).

\section{Discussion}

This is the first study to establish the representative prescribing practices of ADHD drugs in Japan. The prevalence of ADHD drug use in children and adolescents in Japan $(0.4 \%)$ is much lower than that in the USA (5.3\%) (Burcu et al. 2016) and Norway (1.4\%) (Furu et al. 2017), while it is similar to that in Italy (0.2\%) (Piovani et al. 2016), France (0.2\%) (Kovess et al. 2015) and the UK $(0.5 \%)$ (Beau-Lejdstrom et al. 2016). As in Japan, the countries with a similar prevalence have some restriction policies for prescribing ADHD drugs. In Italy, ATX and IR-MPH can be initiated only by specialists with expertise in ADHD treatment after a standardised diagnostic process (Piovani et al. 2016). These drugs can be re-filled by a general practitioner; however, specialists must compile an individual therapeutic plan that contains all the details on dosage and duration of therapy. In France, IR-MPH and OROS-MPH must be initiated by specialists such as child psychiatrists (Kovess et al. 2015). In the UK, ATX, dexamphetamine, guanfacine, lisdexamfetamine, IR-MPH and OROS-MPH are recommended to be initiated only by professionals with expertise in ADHD treatment (National Institute for Health \& Care Excellence, 2006). Such a restriction policy may contribute to a relatively low prevalence of ADHD drug use in the population.

Our results indicate that children and adolescents with ADHD may be currently undermedicated in Japan. The National Institute for Health and Care Excellence guidelines recommend that pharmacological treatment should be initiated when ADHD symptoms are persistent and still causing significant impairment in at least one domain every day despite the implementation and review of environmental modifications (National Institute for Health \& Care Excellence, 2018). The Regional ADHD Registry in Italy found that $44 \%$ of children and adolescents with ADHD had a severe impairment as indicated by a Clinical Global Impressions-Severity score of 5 or higher (Bonati et al. in press). Given the ADHD prevalence of 3.4-7.2\% (Polanczyk et al. 2015; Thomas et al. 2015) and the ratio of severely impaired ADHD (Bonati et al. in press), 1.5-3.2\% of children and adolescents would benefit from pharmacological treatment.

Among ADHD drug users, the percentage of methylphenidate use is much lower in Japan $(64 \%)$ 
Table 2. Prevalence, incidence and persistence of ADHD drug users in children and adolescents

\begin{tabular}{|c|c|c|c|c|c|c|c|}
\hline \multirow[b]{2}{*}{$\begin{array}{l}\text { Sex-age group } \\
\text { (years) }\end{array}$} & \multirow[b]{2}{*}{$\begin{array}{l}\text { No. of } \\
\text { inhabitants } \\
\text { in thousands }\end{array}$} & \multicolumn{2}{|c|}{ Prevalence } & \multicolumn{2}{|c|}{ Incidence } & \multicolumn{2}{|r|}{ Persistence } \\
\hline & & $n$ & $\begin{array}{l}\text { Annual rate } \\
\text { per } 1000 \\
\text { inhabitants } \\
(95 \% \mathrm{CI})\end{array}$ & $n$ & $\begin{array}{l}\text { Annual rate } \\
\text { per } 1000 \\
\text { inhabitants } \\
(95 \% \mathrm{CI})\end{array}$ & $n$ & $\begin{array}{c}\text { Percentage of } \\
\text { 150-day persistence } \\
\text { per } 100 \text { incident } \\
\text { users }(95 \% \mathrm{CI})\end{array}$ \\
\hline Total & 21001 & 86756 & $4.1(4.1-4.2)$ & 30449 & $1.4(1.4-1.5)$ & 18528 & $60.8(60.3-61.4)$ \\
\hline $0-6$ & 7331 & 2659 & $0.4(0.3-0.4)$ & 2382 & $0.3(0.3-0.3)$ & 1523 & $63.9(62.0-65.9)$ \\
\hline $7-12$ & 6559 & 52626 & $8.0(8.0-8.1)$ & 19494 & $3.0(2.9-3.0)$ & 12604 & $64.7(64.0-65.3)$ \\
\hline $13-15$ & 3524 & 22265 & $6.3(6.2-6.4)$ & 6051 & $1.7(1.7-1.8)$ & 3322 & $54.9(53.6-56.2)$ \\
\hline $16-18$ & 3587 & 9206 & $2.6(2.5-2.6)$ & 2522 & $0.7(0.7-0.7)$ & 1079 & $42.8(40.8-44.7)$ \\
\hline Boys & 10755 & 72520 & $6.7(6.7-6.8)$ & 24528 & $2.3(2.3-2.3)$ & 15282 & $62.3(61.7-62.9)$ \\
\hline $0-6$ & 3756 & 2262 & $0.6(0.6-0.6)$ & 2032 & $0.5(0.5-0.6)$ & 1311 & $64.5(62.4-66.6)$ \\
\hline $7-12$ & 3357 & 44961 & $13.4(13.3-13.5)$ & 16235 & $4.8(4.8-4.9)$ & 10691 & $65.9(65.1-66.6)$ \\
\hline $13-15$ & 1806 & 18459 & $10.2(10.1-10.4)$ & 4618 & $2.6(2.5-2.6)$ & 2582 & $55.9(54.5-57.4)$ \\
\hline $16-18$ & 1836 & 6838 & $3.7(3.6-3.8)$ & 1643 & $0.9(0.9-0.9)$ & 698 & $42.5(40.1-44.9)$ \\
\hline Girls & 10244 & 14236 & $1.4(1.4-1.4)$ & 5921 & $0.6(0.6-0.6)$ & 3246 & $54.8(53.5-56.1)$ \\
\hline $0-6$ & 3574 & 397 & $0.1(0.1-0.1)$ & 350 & $0.1(0.1-0.1)$ & 212 & $60.6(55.2-65.7)$ \\
\hline $7-12$ & 3200 & 7665 & $2.4(2.3-2.4)$ & 3259 & $1.0(1.0-1.1)$ & 1913 & $58.7(57.0-60.4)$ \\
\hline $13-15$ & 1718 & 3806 & $2.2(2.1-2.3)$ & 1433 & $0.8(0.8-0.9)$ & 740 & $51.6(49.0-54.3)$ \\
\hline $16-18$ & 1752 & 2368 & $1.4(1.3-1.4)$ & 879 & $0.5(0.5-0.5)$ & 381 & $43.3(40.0-46.7)$ \\
\hline
\end{tabular}

ADHD, attention-deficit/hyperactivity disorder; CI, confidence interval.

than in the UK (94\%) (Beau-Lejdstrom et al. 2016), Norway (94\%) (Furu et al. 2017) and Germany (75$100 \%$ ) (Bachmann et al. 2017). This may be partly explained by the fact that the restriction policy for prescribing ADHD drugs in Japan is only applied to OROS-MPH. For instance, ATX can be prescribed by any physician in Japan. This imbalance in the restriction policy may contribute to a decrease in the use of OROS-MPH. Other explanations for the low prevalence of methylphenidate prescriptions are that IR-MPH is not approved for ADHD treatment in Japan and that both ATX and OROS-MPH are

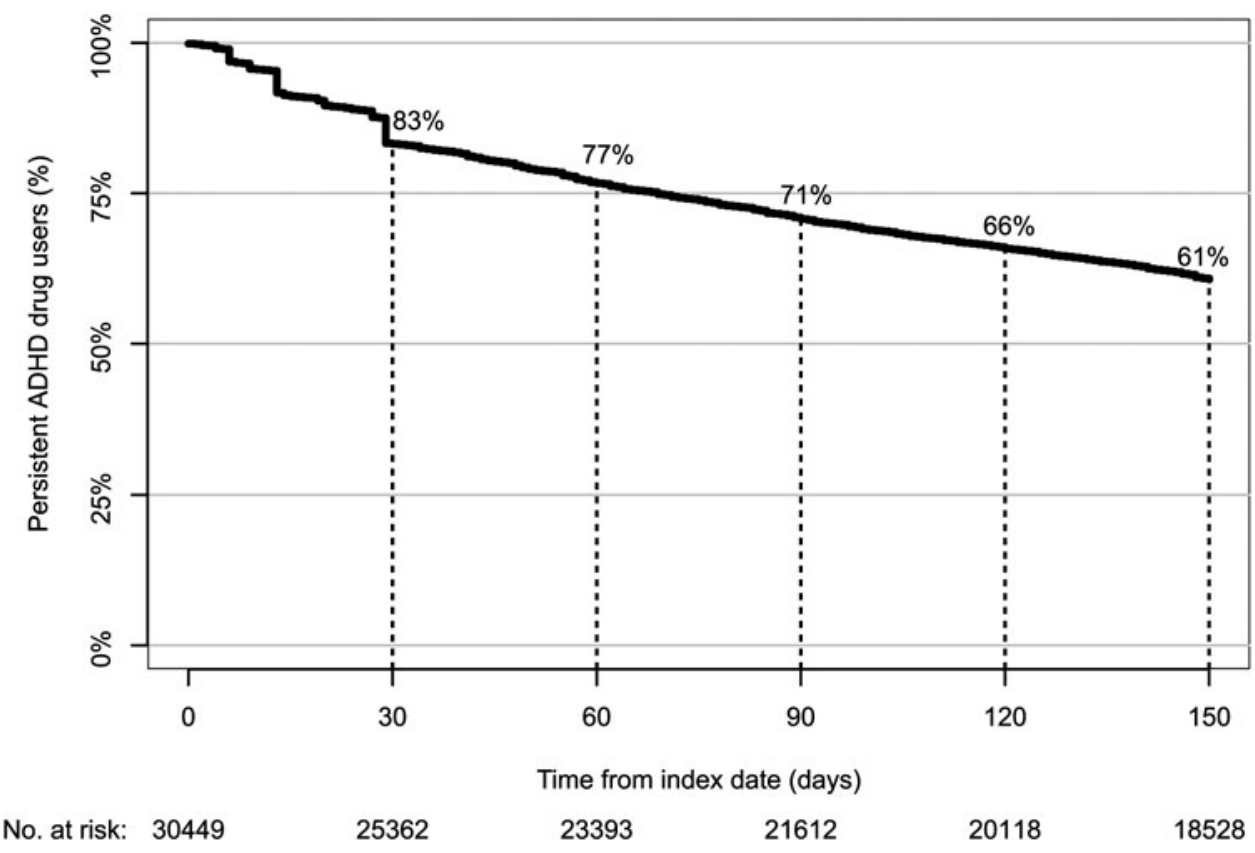

Fig. 1. Percentage of persistent ADHD drug users. 
considered as the first-line drugs for ADHD treatment in the Japanese clinical practice guideline (Saito, 2016).

In addition, the persistence rate of ADHD drug use is much higher in Japan $(61 \%)$ than that in the USA (10-29\% at 150 days) (Lawson et al. 2012), while it is similar to that in the UK $(66 \%$ at 1 year) according to the study conducted by Beau-Lejdstrom et al. who used a threefold wider grace period (Beau-Lejdstrom et al. 2016). We also observed a substantially lower persistence of ADHD drugs among patients who started taking drugs at an older age. These findings were consistent with those of previous studies (Beau-Lejdstrom et al. 2016; Wang et al. 2016). Future research should clarify the reason for early cessation of ADHD drugs, particularly focusing on high school grades.

The main limitation of this study is that the entire population was not accounted for in the database, which comprised $1-2 \%$ of all inhabitants. Nevertheless, our study provides representative evidence on the treatment pattern of ADHD drug use in children and adolescents in Japan.

\section{Acknowledgements}

We are grateful to Dr Daniele Piovani, Dr Raphaelle Beau-Lejdstrom and Dr Thierry Trenque for providing us with information on the restriction policies for ADHD drugs. We would like to thank Editage (www.editage.jp) for English language editing.

\section{Financial Support}

This work was supported by a grant from the Japan Society for the Promotion of Science (number: 18K09991).

\section{Conflict of Interest}

During the past 3 years, $\mathrm{YO}$ received personal fees from Merck \& Co., Inc.; Janssen Pharmaceuticals Inc.; Medical Technology Association; Cando Inc.; and the Japan Medical Data Center. YO has also received research grants from the Japan Agency for Medical Research and Development; Ministry of Health, Labour and Welfare; Japan Society for the Promotion of Science; Institute for Health Economics and Policy; and Mental Health and Morita Therapy. MU received personal fees from Janssen Pharmaceutical Inc.; and Eli Lilly Japan. MU has also received grants from the National Center for Global Health and Medicine; and the Agency for Medical Research and Development. TO received personal fees from Janssen Pharmaceutical Inc.; Eli Lilly Japan; Shionogi \& Co.
Ltd.; Shire Japan Co. Ltd.; Otsuka Pharmaceutical Company; Meiji Seika Pharma Co., Ltd.; Taisho Pharmaceutical Co., Ltd.; Mochida Pharmaceutical Co., Ltd.; Yoshitomiyakuhin Corporation; Chugai Pharmaceutical Co., Ltd.; Eisai Co., Ltd.; MSD K.K.; Novartis Pharmaceutical Company; Sumitomo Dainippon Pharma Co., Ltd.; Mitsubishi Tanabe Pharmaceutical Company; and Kyowa Hakko Kirin Co., Ltd. TO also has received research grants Otsuka Pharmaceutical Company; Ministry of Health, Labour and Welfare; Ministry of Education, Culture, Sports, Science and Technology; Agency for Medical Research and Development; and the Japan Society for the Promotion of Science. TS received personal fees from Eli Lilly Japan; Janssen Pharmaceuticals Inc.; Otsuka Pharmaceutical Company; Sumitomo Dainippon Pharma; Shionogi \& Co. Ltd.; and Shire Japan Co. Ltd. HN received personal fees from Janssen Pharmaceutical Inc.; Eli Lilly Japan; Shionogi \& Co. Ltd.; Shire Japan Co. Ltd.; and Otsuka Pharmaceutical Company. NT received personal fees from Janssen Pharmaceuticals Inc.; Sumitomo Dainippon Pharma; Mitsubishi Tanabe Pharmaceutical Company; Yoshitomiyakuhin Corporation; GlaxoSmithKline plc; Kyowa Hakko Kirin Co., Ltd. NT received personal fees Janssen Pharmaceuticals Inc.; Sumitomo Dainippon Pharma Co., Ltd.; Mitsubishi Tanabe Pharmaceutical Company; Yoshitomiyakuhin Corporation; GlaxoSmithKline plc; and Kyowa Hakko Kirin Co., Ltd. JF has nothing to disclose. JI received personal fees from Janssen Pharmaceuticals Inc.; Eli Lily Japan; Shionogi \& Co. Ltd.; Shire Japan Co. Ltd.; Otsuka Pharmaceutical Company; Mochida Pharmaceutical Co., Ltd.; MSD K.K.; Sumitomo Dainippon Pharma Co., Ltd. JI also has received grants from the Ministry of Education, Culture, Sports, Science and Technology; Ministry of Health, Labour and Welfare; and the Agency for Medical Research and Development.

\section{Ethical Standards}

The local institutional review board at the Institute for Health Economics and Policy approved our study protocol. The review board waived the requirement for informed consent due to the anonymous nature of data.

Y. Okumura ${ }^{12^{*}}$, M. Usami ${ }^{3}$, T. Okada ${ }^{4}$, T. Saito ${ }^{5}$, H. Negoro ${ }^{6}$, N. Tsujiii ${ }^{7}$ J. Fujita ${ }^{8}$ and J. Iida ${ }^{9}$

${ }^{1}$ Research Department, Institute for Health Economics and Policy, Association for Health Economics Research and Social Insurance and Welfare, Tokyo, Japan

${ }^{2}$ Department of Psychiatry and Behavioral Science, Tokyo Metropolitan Institute of Medical Science, Tokyo, Japan 
${ }^{3}$ Department of Child and Adolescent Psychiatry, Kohnodai Hospital, National Center for Global Health and Medicine, Chiba, Japan

${ }^{4}$ Department of Child and Adolescent Psychiatry, Nagoya University Graduate School of Medicine, Aichi, Japan ${ }^{5}$ Department of Child and Adolescent Psychiatry, Graduate School of Medicine, Hokkaido University, Hokkaido, Japan ${ }^{6}$ Graduate School of Professional Development in Education, Nara University of Education, Nara, Japan ${ }^{7}$ Department of Neuropsychiatry, Kindai University Faculty of Medicine, Osaka, Japan

${ }^{8}$ Department of Child Psychiatry, Yokohama City University Hospital, Kanagawa, Japan ${ }^{9}$ Department of Human Development, Faculty of Nursing, Nara Medical University, Nara, Japan

\section{References}

Bachmann CJ, Philipsen A, Hoffmann F (2017). ADHD in Germany: trends in diagnosis and pharmacotherapy.

Deutsches Ärzteblatt International 114, 141-148.

Beau-Lejdstrom R, Douglas I, Evans SJ, Smeeth L (2016). Latest trends in ADHD drug prescribing patterns in children in the UK: prevalence, incidence and persistence. BMJ Open 6, e010508.

Bonati M, Reale L, Zanetti M, Cartabia M, Fortinguerra F, Capovilla G, Chiappedi M, Costantino A, Effedri P, Luoni C, Martinelli O, Molteni M, Ottolini A, Saccani M, Lombardy AG (in press). A regional ADHD center-based network project for the diagnosis and treatment of children and adolescents with ADHD. Journal of Attention Disorders.

Burcu M, Zito JM, Metcalfe L, Underwood H, Safer DJ (2016). Trends in stimulant medication use in commercially insured youths and adults, 2010-2014. JAMA Psychiatry 73, 992-993.

Furu K, Karlstad O, Zoega H, Martikainen JE, Bahmanyar S, Kieler H, Pottegard A (2017). Utilization of stimulants and atomoxetine for attention-deficit/hyperactivity disorder among 5.4 million children using population-based longitudinal data. Basic $\mathcal{E}$ Clinical Pharmacology $\mathcal{E}$ Toxicology 120, 373-379.

Kovess V, Choppin S, Gao F, Pivette M, Husky M, Leray E (2015). Psychotropic medication use in French children and adolescents. Journal of Child and Adolescent Psychopharmacology 25, 168-175.

Kubo S, Noda T, Myojin T, Nishioka Y, Higashino T, Matsui H, Kato G, Imamura T (2018). National Database of Health Insurance Claims and Specific Health Checkups of Japan (NDB): Outline and patient-matching technique. https://www.biorxiv.org/content/biorxiv/early/2018/03/29/ 280008.full.pdf (accessed 10 April 2018).
Lawson KA, Johnsrud M, Hodgkins P, Sasane R, Crismon ML (2012). Utilization patterns of stimulants in ADHD in the Medicaid population: a retrospective analysis of data from the Texas Medicaid program. Clinical Therapeutics 34, 944-956, e4.

Ministry of Health, Labour and Welfare (2013). On the use of the National Database of Health Insurance Claim Information and Specified Medical Checkups (in Japanese). http://www.mhlw.go.jp/stf/shingi/2r9852000002ss9z-att/ 2r9852000002ssfg.pdf. Accessed 4 December 2017.

Ministry of Internal Affairs and Communications (2015). Population Estimates in 2014: Table 1: Population by Age (Single Year), Sex and Sex ratio (in Japanese). https://www. e-stat.go.jp/stat-search/files?page=1\&layout=datalist \&stat_infid $=000029025950 \&$ lid $=000001132435$. Accessed 1 January 2018.

National Institute for Health and Care Excellence (2006). Methylphenidate, Atomoxetine and Dexamfetamine for Attention Deficit Hyperactivity Disorder (ADHD) in Children and Adolescents. British Psychological Society: Leicester.

National Institute for Health and Care Excellence (2018). Attention Deficit Hyperactivity Disorder: Diagnosis and Management. British Psychological Society: Leicester.

Okumura Y, Sakata N, Takahashi K, Nishi D, Tachimori H (2017). Epidemiology of overdose episodes from the period prior to hospitalization for drug poisoning until discharge in Japan: an exploratory descriptive study using a nationwide claims database. Journal of Epidemiology 27, 373-380.

Palli SR, Kamble PS, Chen H, Aparasu RR (2012). Persistence of stimulants in children and adolescents with attention-deficit/hyperactivity disorder. Journal of Child and Adolescent Psychopharmacology 22, 139-148.

Piovani D, Clavenna A, Cartabia M, Bonati M (2016). Psychotropic medicine prescriptions in Italian youths: a multiregional study. European Child $\mathcal{E}$ Adolescent Psychiatry 25, 235-245.

Polanczyk GV, Salum GA, Sugaya LS, Caye A, Rohde LA (2015). Annual research review: a meta-analysis of the worldwide prevalence of mental disorders in children and adolescents. Journal of Child Psychology and Psychiatry, and Allied Disciplines 56, 345-365.

Saito M (2016). Diagnosis and Management for ADHD: Clinical Practice Guideline (in Japanese). Jiho: Tokyo.

Thomas R, Sanders S, Doust J, Beller E, Glasziou P (2015). Prevalence of attention-deficit/hyperactivity disorder: a systematic review and meta-analysis. Pediatrics 135, e994e1001.

Wang LJ, Yang KC, Lee SY, Yang CJ, Huang TS, Lee TL, Yuan SS, Shyu YC (2016). Initiation and persistence of pharmacotherapy for youths with attention deficit hyperactivity disorder in Taiwan. PLoS ONE 11, e0161061.

Wang LJ, Lee SY, Yuan SS, Yang CJ, Yang KC, Huang TS, Chou WJ, Chou MC, Lee MJ, Lee TL, Shyu YC (2017). Prevalence rates of youths diagnosed with and medicated for ADHD in a nationwide survey in Taiwan from 2000 to 2011. Epidemiology and Psychiatric Sciences 26, 624-634. 